\title{
FUNDAMENTAL STUDIES WITH A MONODISPERSE AEROSOL-BASED LIQUID CHROMATOGRAPHY/MASS SPECTROMETRY INTERFACE (MAGIC-LC/MS)
}

\author{
Final Progress Report \\ December 1, 1989 - December 31, 1992 \\ Richard F. Browner \\ School of Chemistry and Biochemistry \\ Georgia Institute of Technology \\ Atlanta, GA 30332-0400
}

DISCLAIMER

\begin{abstract}
This report was prepared as an account of work sponsored by an agency of the United States Government. Neither the United States Government nor any agency thereof, nor any of their employees, makes any warranty, express or implied, or assumes any legal liability or responsibility for the accuracy, completeness, or usefulness of any information, apparatus, product, or process disclosed, or represents that its use would not infringe privately owned rights. Reference herein to any specific commercial product, process, or service by trade name, trademark, manufacturer, or otherwise does not necessarily constitute or imply its endorsement, recommendation, or favoring by the United States Government or any agency thereof. The views and opinions of authors expressed herein do not necessarily state or reflect those of the United States Government or any agency thereof.
\end{abstract}

December 1992

PREPARED FOR THE U.S. DEPARTMENT OF ENERGY

UNDER GRANT NUMBER DE-FG05-85ER13435 


\section{ACCOMPLISHMENTS DURING PERIOD DECEMBER 1, 1989}

TO DECEMBER 31, 1992

One of the most dramatic developments in mass spectrometry in the last fifteen years has been the evolution of versatile and powerful interfacing approaches that allow direct chromatographic coupling of separations techniques to mass spectrometers. Separations techniques successfully coupled include normal bore and micro-bore reversed phase chromatography and more recently, capillary electrophoresis. The most successful of these approaches have been aerosol-based. Aerosols provide a unique means of converting column effluent into a form that allows ion production in the mass spectrometer. At the present time, the dominant forms of chromatographic interfacing are Thermospray (TSP), Particle Beam (PB) and Electrospray (ES). Each of these techniques compliments the others, and together they have become a rapidly growing field with wide practical applications in environmental, biomedical and energy-related problems of analysis.

This report describes the research carried out under DOE support directed toward fundamental studies with the Particle Beam LC/MS interface (also know as the MAGIC LC/MS interface). The primary goal has been to gain a better understanding of aerosol generation, transport, vaporization, and ionization processes which forms the basis of the technique. Gaining a deeper understanding of the basic physical processes on which particle Beam LC/MS is based provides the most direct way to improve performance benchmarks, such as (1) detection limits (2) quality of mass spectra (3) range of compound types possible, and (4) the ability to interface with all types of separation technique.

Although on-line LC interfacing techniques have been available since the pioneering direct liquid introduction (DLI) work of McLafferty in 1974, it is only really in the last fourteen years that LC/MS has experienced significant growth, following the introduction of Thermospray interfacing (Vestal et al., 1978). In the last few years the pace of growth has accelerated considerably, since the introduction of Particle Beam LC/MS in 1984 (PB-LC/MS) (Willoughby et al.) and Electrospray (ES/MS) in 1989 (Fenn et al.). PB-LC/MS and ES/MS have been rapidly adopted and have achieved remarkable growth in industrial and governmental laboratories. Numerous conference presentations and research publications have followed, and these techniques are now being used to support much forefront biomedical and environmental research.

In light of this rapid growth, our research effort has been devoted to developing a fundamental understanding of the basic physical process which underlie aerosol mass spectrometry interfacing approaches.

\section{Chromatographic Peak Broadening Studies}

Particle beam LC/MS interfacing allows mass spectrometry to be coupled to liquid chromatography separations using a wide range of solvents, with relatively little change necessary from normal LC operating conditions. Although there are some slight response variations when different solvents are used, solvent flows in the range 0.3 to $0.5 \mathrm{~mL} / \mathrm{min}$ give optimum results. Measurement of external variance produced by the interface shows peak broadening to be negligible for normal bore ( $4.6 \mathrm{~mm}$ i.d.) packed columns, but to be detectable, though relatively slight, for small bore $(2.1 \mathrm{~mm}$ i.d.) packed columns. Microbore $(\leq 1 \mathrm{~mm}$ i.d.) columns are unsuitable for present systems both because the liquid flow is inadequate for efficient aerosol generation without addition of a makeup stream, and because excessive band broadening occurs in the interface. The use of solvent gradients causes no significant change in the quality of mass spectra produced by electron impact or chemical ionization. However, the use of $100 \%$ water is 
found to cause a decrease in response of approximately a factor of 2 compared to other solvents. Separations may readily be carried out in buffer solutions, but buffers must be volatile (e.g. $\mathrm{CH}_{3} \mathrm{COOH} / \mathrm{NH}_{4} \mathrm{COOCH}_{3}$ ) in order to avoid contamination of the ion source and possible blockage of the momentum separator

\section{Gradient Elution.}

Many separation problems in liquid chromatography require gradient elution for adequate resolution. The ability of an interface to work readily with gradients is therefore a very important feature in LC/MS. However, gradient elution has often proved to be a major obstacle in the design of working interfaces. Generally, the problem has arisen because a change in properties of the solvent influences either the aerosol formation process, as with Thermospray, or the ionization process, as with DLI.

LC/MS interfaces in which solvent participates in the ionization process will be affected by solvent composition. For instance in Thermospray LC/MS, operation with gradient elution is only practical with inverse temperature programming of the Thermospray source [1] in order to maintain vaporization of a constant fraction of the solvent. The fraction of solvent vaporized is related to the specific heat of the solvent, and so will vary as the mobile phase composition changes. The nature of Thermospray spectra also changes as the water content of the mobile phase is reduced. Spectra which consist initially primarily of $(\mathrm{M}+\mathrm{H})^{+}$ions gain an increasing fraction of $(M+\text { solvent })^{+}$ions as the organic content of the solvent rises.

Solvent elimination interfaces, such as the moving-belt interface, do not appear to experience as much difficulty with gradient separations as do DLI and Thermospray. However, difficulty has been reported in working with high water content solvents at the initiation of a reversed-phase gradient separation [2].

\section{Quality of Mass Spectra Observed with Different Solvents.}

In spite of the $2 \mathrm{x}$ signal loss found with water, the quality of the EI mass spectra generated with both types of particle beam interfaces showed no detectable differences. Electron impact mass spectra generated for caffeine $\mathrm{C}_{8} \mathrm{H}_{10} \mathrm{~N}_{4} \mathrm{O}_{2}$ and thiamine, using the both types of particle beam interface and thiamine, and using three solvents of different composition: (1) $100 \%$ methanol (2) $50 \%$ methanol $/ 50 \%$ water and (3) $100 \%$ water appear identical in all important respects. They all gave comparable molecular ions at m/z 194 and $\mathrm{m} / \mathrm{z} 264$ respectively, structurally meaningful fragmentation patterns, and correspond closely to the EPA/NIH reference spectra. It is also noteworthy that no solvent cluster ions were observed in any of the spectra obtained with either of the particle beam interfaces, irrespective of solvent composition.

\section{Use of Buffer Solutions.}

All practical methods of LC/MS interfacing developed to date require the use of volatile buffers. The particle beam LC/MS interface is no different in this regard, and for much the same reasons as those found with the other systems. The use of involatile buffers, such as phosphate buffers, with the interface results in the presence of an involatile residue which will pass into the mass spectrometer, and rapidly lead to serious contamination of the ion source. Additionally, if high concentrations of such buffers are used, there will be some build up of material around the skimmers in the momentum separator, which in time can lead to partial or total blockage of the orifices.

Particle beam interfaces work well with volatile buffers, and a number of separations have been carried out using ammonium acetate/acetic acid mixtures. The performance of the interface 
when used with these buffers appears to be largely unaffected by their presence in terms of both system response and quality of mass spectra generated.

External Variance Measurements.

Adequate chromatographic resolution between adjacent peaks is clearly an important criterion in any LC/MS interface, and is related directly to the peak variance. In a well designed system, the width of the final bands should be due almost entirely to intra-column band broadening, resulting from eddy diffusion, longitudinal diffusion and mass transfer processes taking place on the column.

In a conventional LC system, band broadening external to the column, or external variance, may result from a variety of sources, including an increase in volume between the injector and the detector and an increase in the sample volume injected. Common sources of external variance include large diameter or extra lengths of tubing, fittings which do not meet zero-dead volume requirements, and detector flow cells. In addition, the detector electronics and recording systems may contribute to the overall external variance [3].

The processes which may lead to band broadening in the particle beam type interfaces are more complex than in a traditional liquid chromatograph equipped with a flow cell detector. First, an eluting band must exit the fused silica capillary and form an aerosol which will travel to the ion source of the mass spectrometer in a stream of helium gas. Then, the analyte particles contained in the band passing through the interface must vaporize off the walls of the ion source. The analyte gas phase components will then diffuse back into the electron beam, where they will be ionized and ultimately travel to the detector.

In conventional liquid chromatography, liquid dispersion in the tubing is a consequence of a parabolic velocity profile across the tubing and turbulent rather than laminar flow [4]. Particle beam interfaces are aerosol-based and the peak dispersion should be minimal as long as the flow is laminar and not turbulent. The velocity profile should largely be eliminated because less drag will occur against the gas phase and in partial vacuums than along a stainless steel surface of the tubing. In addition, dispersion will be reduced because the sample band will have reduced residence time as compared to a sample passing through the same length of tubing [5]. It appears from the above discussion that the main site for band broadening should occur in the ion source region at the point of final particle vaporization.

Attention to external variance is particularly important in particle beam LC/MS because of the optimum flow rates of the interface, which match small bore and fast liquid chromatography columns in a nearly ideal manner. These columns place high demands on the liquid chromatography system as they necessitate low external variances in the total system. Visual comparisons between HPLC chromatograms obtained with a UV detector and the total ion chromatogram from the mass spectrometer are often quoted in the literature. This procedure, however, lacks any reliable quantitation which can be used to compare various systems.

\section{Interface Performance.}

A series of total ion chromatograms were obtained for a test mixture of carboxylic acids, with retention times measured using the mass spectrometer data station. The total dispersion for each peak was calculated and plotted against the square of the retention time. Table I contains the calculated values for $\sigma_{\text {ext }}^{2}$, the total variance; $r$, the correlation coefficient; and $\sigma_{\text {ext }}$, the external variance. In Table II, the maximum external dispersion allowed in order to maintain chromatographic integrity is listed for four types of columns. A comparison between the values of external variance measured for the particle beam interface and the values in Table II reveal the extent of particle beam's compatibility with various column types. 
Table I. Variance values calculated from linear regression analysis of variance and retention data.

Type \#1 PB Interface

\begin{tabular}{lllll}
\hline $\begin{array}{l}\text { Temperature } \\
\left({ }^{\circ} \mathrm{C}\right)\end{array}$ & $\begin{array}{c}\mathrm{r}^{2}{ }_{\text {ext }} \\
\left(\mathrm{s}^{2}\right)\end{array}$ & $\mathrm{r}$ & & $\mathrm{r}_{\text {ext }}$ \\
& & & & $(\mu \mathrm{L})$ \\
300 & 0.59 & 0.9789 & 3.84 & 10.60 \\
220 & 4.49 & 0.9077 & \\
\hline
\end{tabular}

Type \#2 PB Interface

\begin{tabular}{llll}
\hline $\begin{array}{c}\text { Temperature } \\
\left({ }^{\circ} \mathrm{C}\right)\end{array}$ & $\begin{array}{c}\mathrm{r}^{2}{ }_{\text {ext }} \\
\left(\mathrm{s}^{2}\right)\end{array}$ & $\mathrm{r}$ & $\begin{array}{c}\mathrm{r}_{\text {ext }} \\
(\mu \mathrm{L})\end{array}$ \\
\hline 300 & 0.48 & 0.9970 & 4.62 \\
220 & 3.17 & 0.9616 & 11.86 \\
\hline
\end{tabular}

The maximum allowable external variance for a conventional column is $15 \mu 1$. The values for particle beam at $220^{\circ} \mathrm{C}$ and $300^{\circ} \mathrm{C}$ are $10.60 \mu \mathrm{l}$ and $3.84 \mu \mathrm{l}$ respectively, which are well below the upper limit.

For conventional and fast liquid chromatography columns, the particle beam LC/MS interface system appears to be completely compatible at both $300^{\circ} \mathrm{C}$ and $220^{\circ} \mathrm{C}$, when using the capillary tubing transfer line. The lowest external variance found for the particle beam interface was 3.84 $\mu 1$. This value exceeds slightly the maximum tolerable limit of $3.1 \mu \mathrm{l}$ for small bore columns. The use of these columns with the particle beam interface will therefore result in measurable, if slight, band broadening. The maximum permissible external variance for microbore $(\leq 1 \mathrm{~mm}$ i.d.) columns is $0.7 \mu \mathrm{l}$, which is well below the best external variance value found with particle beam. At present, therefore, use of the particle beam interface with these columns would lead to significant loss of chromatographic resolution. 
Table II. Maximum allowable external dispersion $(\mu \mathrm{L})$ for various columns, defined by $\sigma_{\text {ex }}=0.5 \sigma_{\text {col }}$

\begin{tabular}{|c|c|}
\hline Column type & $k^{\prime}=1[20]^{\sigma_{e x t}}$ \\
\hline $\begin{array}{l}\text { Conventional } \\
4.6 \times 100 \mathrm{~mm} \\
5 \mu \mathrm{m} \text { packing }\end{array}$ & 15.0 \\
\hline $\begin{array}{l}\text { Fast } \\
5.0 \times 60 \mathrm{~mm} \\
3 \mu \mathrm{m} \text { packing }\end{array}$ & 10.6 \\
\hline $\begin{array}{l}\text { Small bore } \\
2.1 \times 100 \mathrm{~mm} \\
5 \mu \mathrm{m} \text { packing }\end{array}$ & 3.1 \\
\hline $\begin{array}{l}\text { Microbore } \\
1 \times 100 \mathrm{~mm} \\
5 \mu \mathrm{m} \text { packing }\end{array}$ & 0.7 \\
\hline
\end{tabular}

The relationship between external variance and ion source temperature for the particle beam interface dmonstrates that dispersion is quite high at low ion source temperatures, but drops to reasonable values at normal ion source operating temperatures. The rate of analyte vaporization from the ion source is an issue of great interest in particle beam LC/MS, as it influences both band breadth and the quality of the mass spectrum produced for thermally labile compounds. The factors involved in these processes are quite complex, and related to both the compound type and the nature of the vaporization surface, in addition to the source temperature. From the perspective of the present study, the need to maintain a source temperature in excess of the minimum value necessary to produce sufficiently rapid analyte vaporization to avoid band broadening is clear. There will be a minimum source temperature necessary to ensure rapid vaporization without band broadening, which will be a direct function of the compound volatility. This factor, which is not normally of importance in GC/MS work, must be taken into account to ensure optimum chromatographic performance.

Dispersion performance of the particle beam interface compares quite favorably with other LC/MS interfaces. The DLI interface has a reported variance of 10-40 $\mu \mathrm{L}$ [6]. Most of this is attributable to the long length of tubing necessary to reach the ion source (at least $40 \mathrm{~cm}$ ) utilized in most commercial DLI systems. The moving-belt interface has a reported value for fluorene at $1 \mathrm{~mL} / \mathrm{min}$ of $15.8 \mu \mathrm{L}$ [7]. The reported variance for the moving-belt interface is compound dependent, however, and significant skewing is reported for polar compounds [8]. The possibility of similar effects occurring with the particle beam interface has not yet been investigated. 


\section{Carrier Effects with the Particle Beam Interface}

A major issue with particle beam LC/MS is the so-called "carrier effect." This describes the influence of concentration on particle size, and hence particle mass, and its influence on the transport efficiency of analyte passing through the interface. In a negative connotation, this can lead directly to non-linearity in the calibration graphs for species. The "carrier effect" is the effect of adding mass to the solvent-free particles through the medium of a high concentration of matrix added to the analyte solution. After solvent evaporation, the more massive particle remaining reaches a higher momentum in the interface, and so has a higher probability of traversing the momentum separator than the matrix-free particle. This increases the analyte transport, and leads to higher analyte signal in the detector.

However, the influence of analyte volatility could be quite significant in influencing the mass, and hence the momentum of the dried particle as it passes through the interface. Consequently, it might be anticipated that for a series of compounds of varying volatility, the more volatile species would tend to have a lower analyte transport efficiency, and so lead to a lower response in the mass spectrometer than a less volatile homologue, on a mass normalized basis. This should also lead to non-linear calibration curves for volatile species, which should become more linear with the addition of less volatile matrix species.

Calibration curves for naphthalene with pyrene carrier are shown in Figure 1.1. Each curve in the figure represents a different amount of carrier added, from zero to $13.5 \mu \mathrm{g}$. Similar curves for anthracene and naphthacene appear in Figures 1.2 and 1.3. (Error bars included in Figure 1.2 represent the greatest RSD observed; RSD's never exceeded 10\%.) After analysis of the data, several trends can be observed. Considering first the calibration curves without carrier, become clear through the generation of analytical curves for a homologous series of compounds, such as the polynuclear aromatics. These are particularly suitable, as they have simple mass spectra, are unreactive and thermally stable, and cover a very wide range of vapor pressures at room temperature. In these experiments, therefore, naphthalene, anthracene, naphthacene and pyrene were used as model compounds. from the range of 200 to $1500 \mathrm{ng}$, it is observed that linearity increases with decreasing volatility (naphthalene $\mathrm{r}^{2}=0.9536$, anthracene ${ }^{2} \mathrm{r}=0.9797$, and naphthacene $\left.r^{2}=0.9896\right)$. The sublimation rate of a solid particle is mainly dependent on two factors: the volatility of the species and the surface area to volume ratio (SA/V). Figure 1.4 below illustrates the effect of increasing injection amount on the surface area to volume ratio. The naphthalene suffers from decreased transport efficiencies at lower concentrations, most likely due to a combination of high volatility and high $S A / V$ ratios. In contrast, while anthracene and naphthacene experience almost identical increases in SA/V ratios at the lower concentrations, their decreased volatility limits sublimation and subsequent losses in the momentum separator. At low injection masses $(<1 \mu \mathrm{g})$, the SA/V ratio increases very rapidly, leading to greatly enhanced sublimation rates for volatile species. 


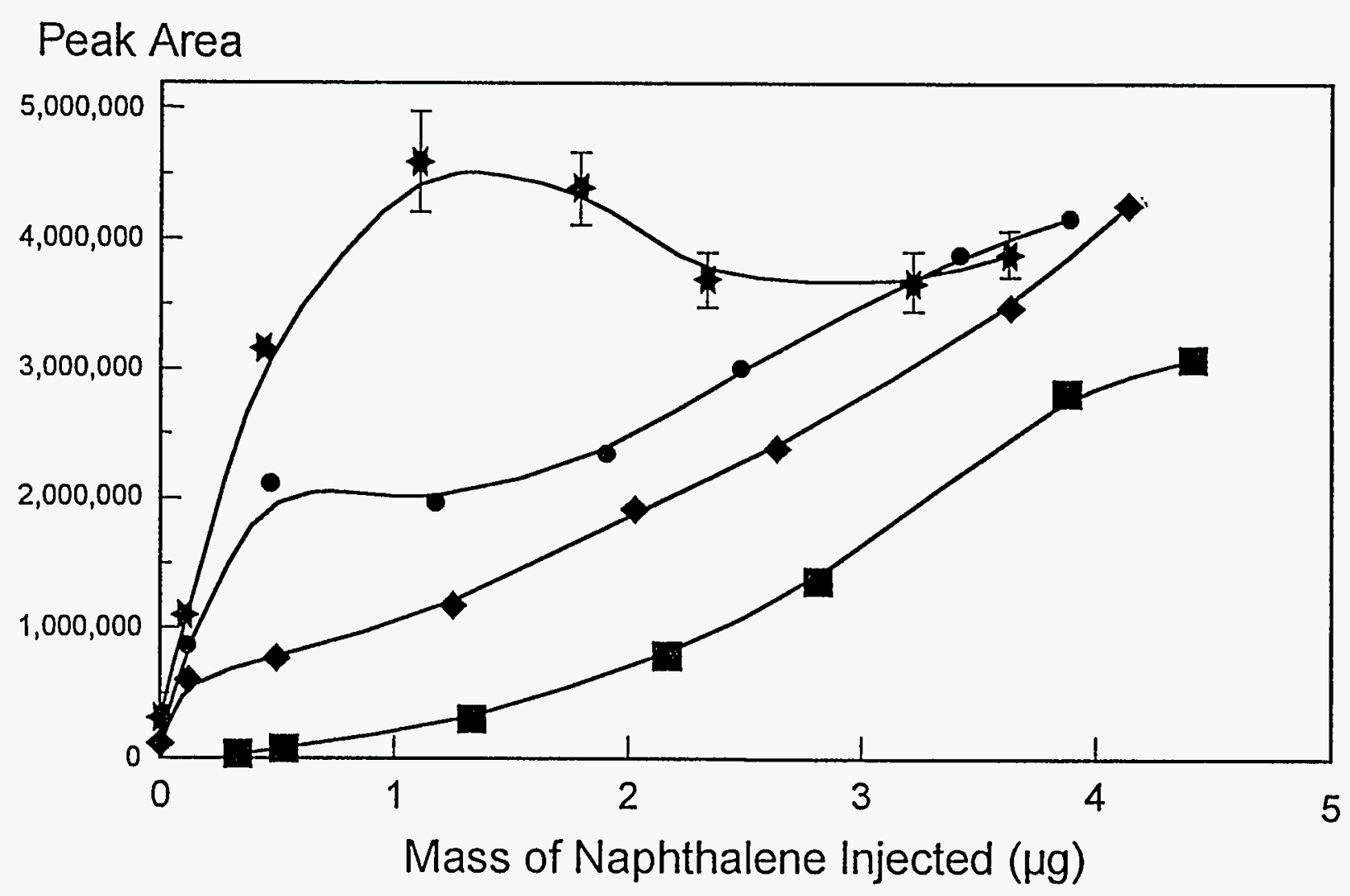

No Carrier $5 \mu \mathrm{g}$ Carrier $9.5 \mu \mathrm{g}$ Carrier $13.5 \mu \mathrm{g}$ Carrier 


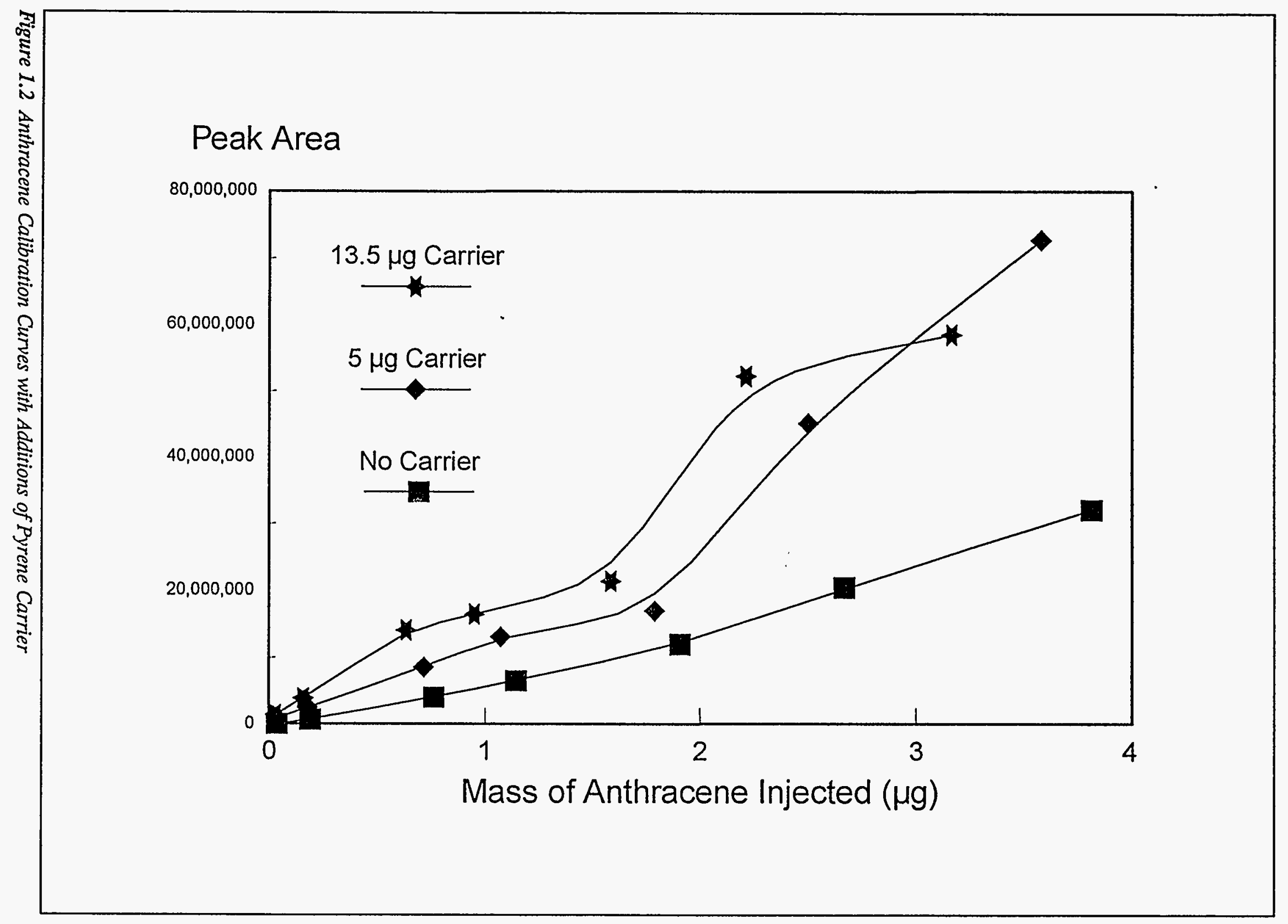




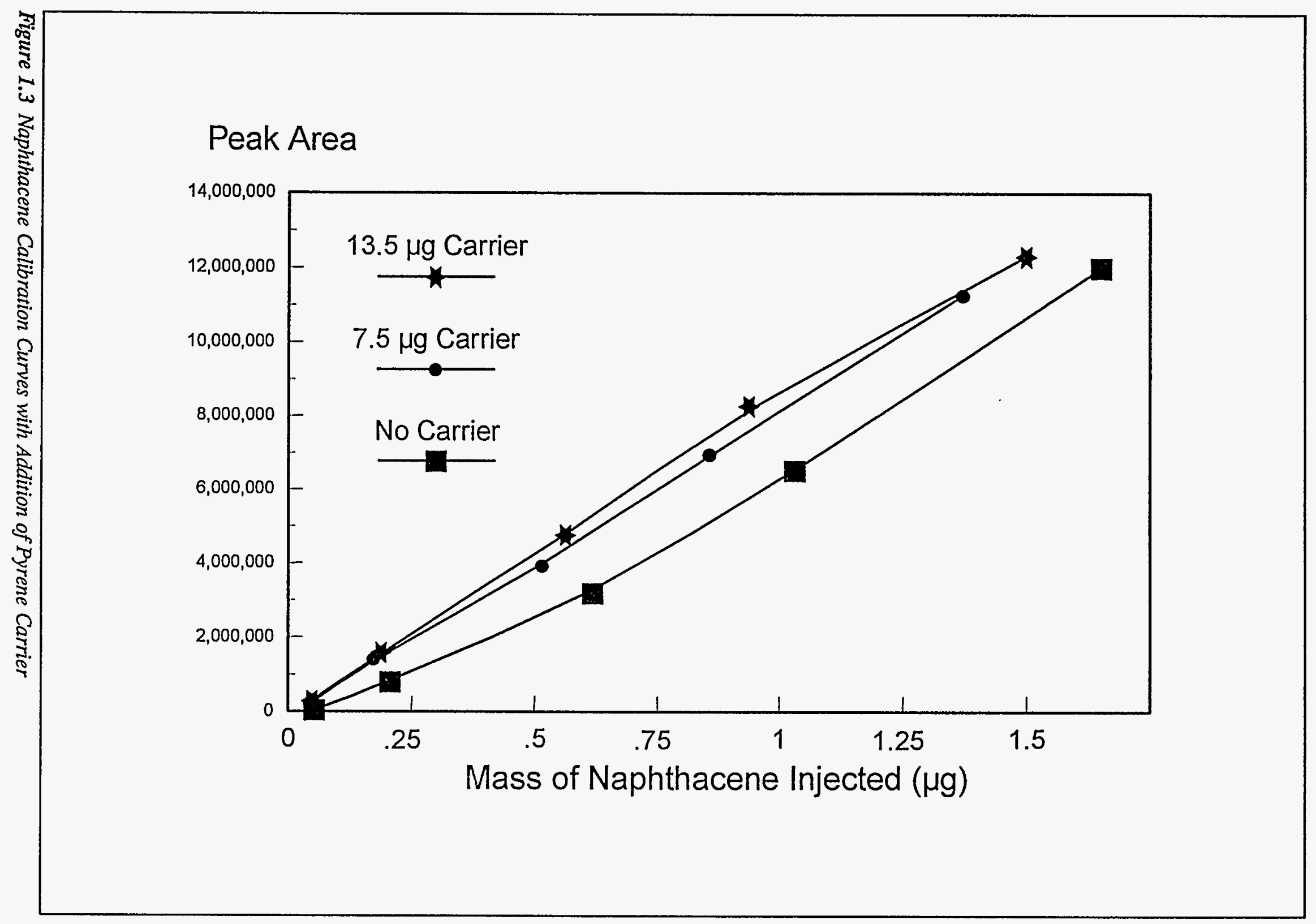




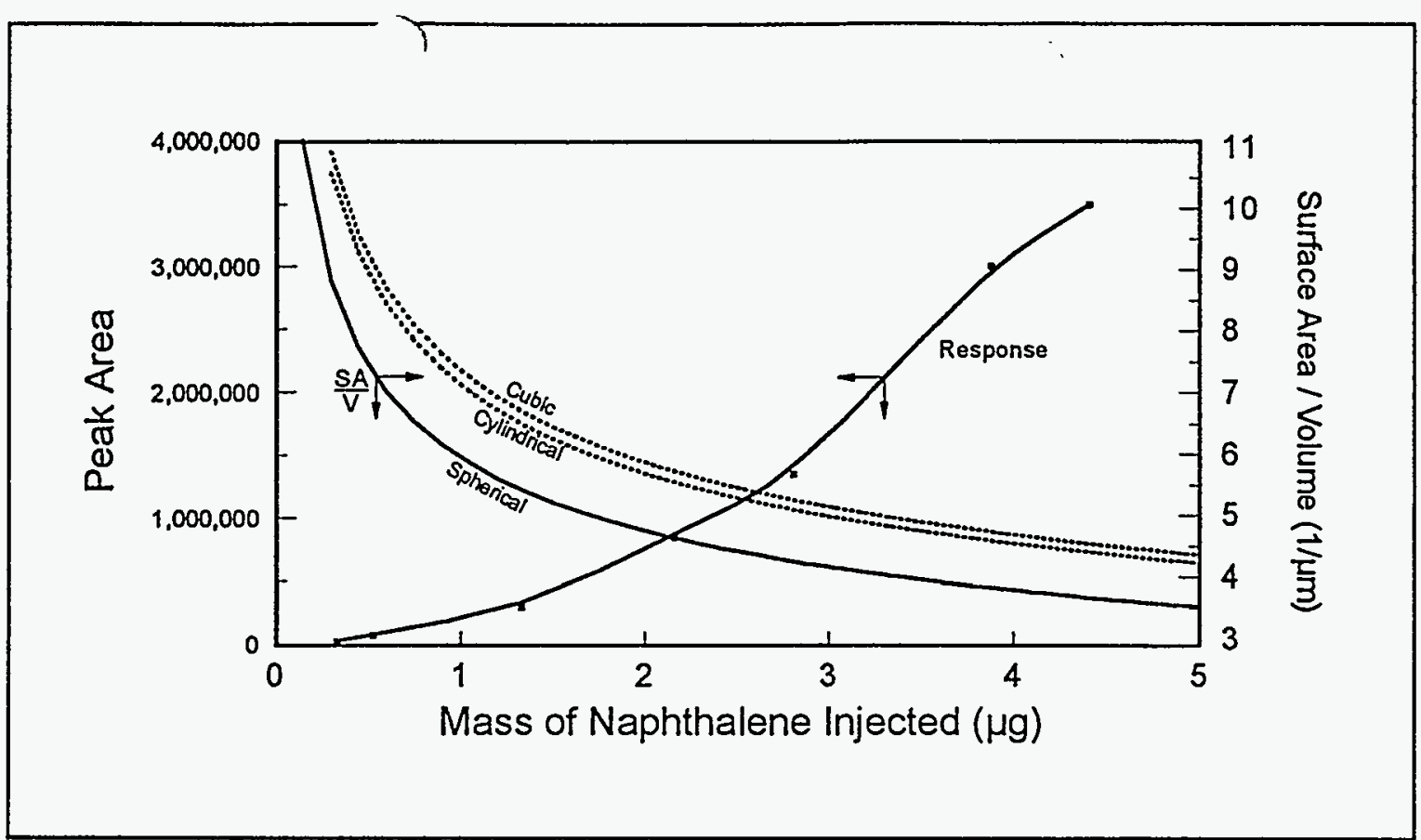

Figure 1.4 Surface Area to Volume Ratio \& MS Response vs. Amount of Naphthalene Injected

Naphthalene, being highly volatile, gave relatively low response per mass injected, with a detection limit of only $200 \mathrm{ng}$ (without carrier). However, after the addition of nonvolatile pyrene carrier, the detection limit decreased by an order of magnitude (20ng with $13.5 \mu \mathrm{g}$ carrier). Anthracene gave a higher response per unit mass than naphthalene, with a pure compound detection limit of 35ng. After the addition of $13.5 \mu \mathrm{g}$ carrier, the detection limit decreased to $5 \mathrm{ng}$. The highly involatile compound naphthacene gave the greatest response per mass injected, with a detection limit of 500pg. In this case the addition of a nonvolatile carrier had almost no effect on the naphthacene response, even at values near the detection limit. Further experiments were carried out over a narrower injected mass range (near the detection limit), and are presented in Figure 1.5. As can be seen, no significant enhancement in signal was observed after the addition of various amounts of pyrene carrier, even down to 500pg. At the 500pg level, the addition of $13.5 \mu \mathrm{g}$ of pyrene carrier should produce particles with approximately 27,000 times greater mass, yet no enhancement in signal was observed. These data seem to discount the existence of a dominant mass transport effect as suggested by many researchers. Under normal operating conditions, the momentum separator separates gas (solvent and other volatiles) from solid (analyte and other nonvolatiles). This corresponds to a difference in momentum of greater than ten orders of magnitude. Knowing this, it seems difficult to believe that doubling the mass of a particle could increase MS signal by any significant amount. Instead, it seems likely that volatility and the SA/V ratio of the particles play the major role during transport through the interface.

The shapes of some of the curves in Figures 1.1,1.2, and 1.3 might initially seem to be rather complex. Clearer trends emerge, however, when the data are presented as in Figure 3.10, which shows a plot of enhancement factor (signal with carrier / signal without carrier) versus mass of analyte injected, for the three analytes. This graph clearly shows that the highly volatile naphthalene is greatly affected by the addition of nonvolatile pyrene. The analyte of medium volatility, anthracene, exhibits a medium signal enhancement, and the nonvolatile naphthacene is almost totally unaffected by the presence of carrier. Furthermore, the enhancements for naphthalene and anthracene become much greater at lower injected mass of analyte. Generally, anthracene would not be considered a compound of medium volatility, especially at temperatures near room temperature. However, the path that the particles travel on their way through the interface involves both reduced pressure and highly turbulent zones. While passing into the 


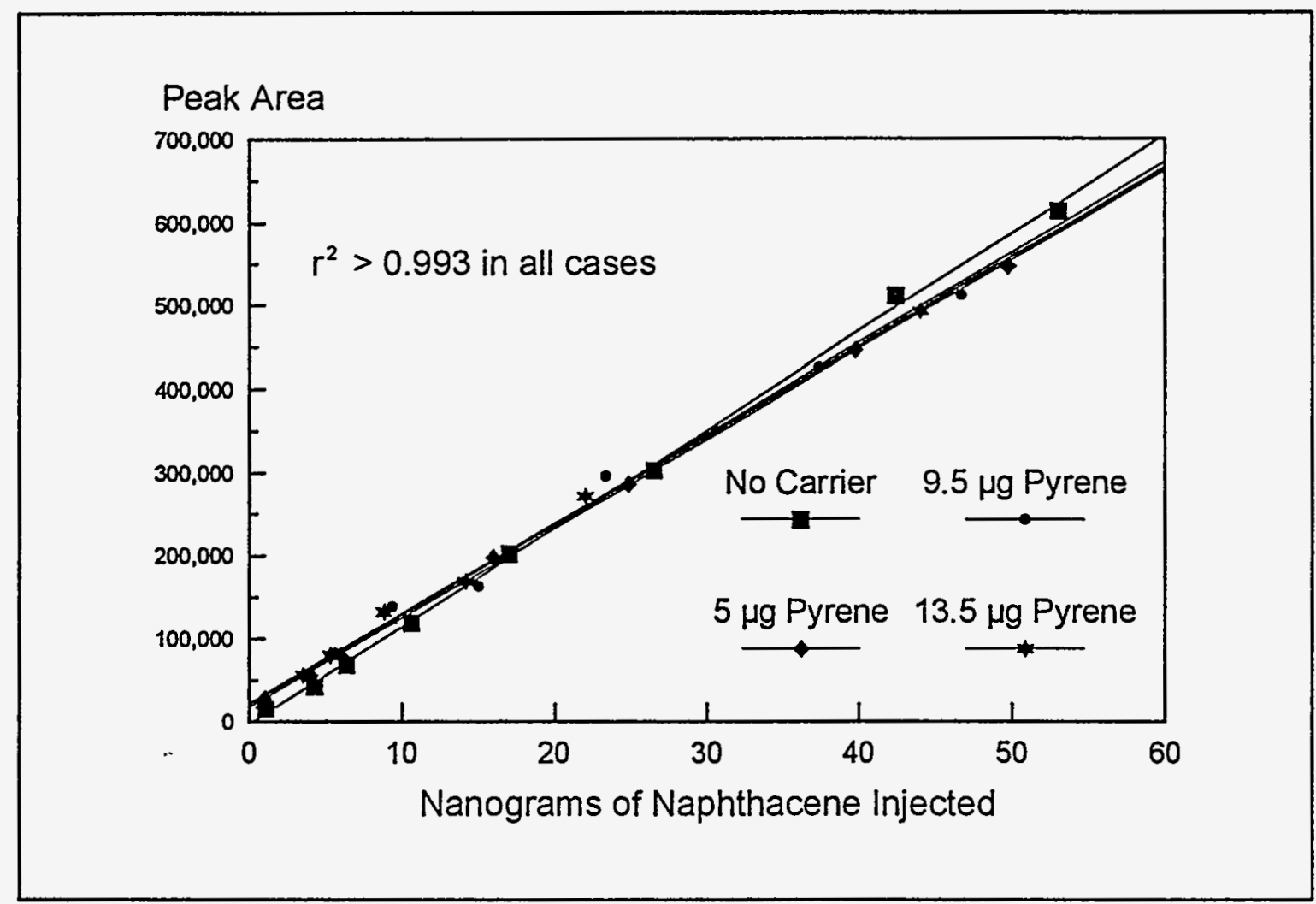

Figure 1.5 Naphthacene with Pyrene Carrier (Near the Detection Limit)

momentum separator, the particles undergo supersonic expansion into a low pressure region, resulting in great turbulence and the formation of a Mach disc. Under these conditions it is not unrealistic to propose that losses due to volatility may occur for anthracene and other compounds of similar volatility.

After examination of Figure 1.1, it is important to note that in the presence of pyrene carrier, as the mass of the naphthalene is increased, the mass of the pyrene remains constant. At low naphthalene concentrations, the pyrene carrier is present in much greater abundance than the naphthalene, resulting in dramatic increases in sensitivity. However, as the mass of the naphthalene increases, the mass of naphthalene in the particle becomes comparable to the mass of pyrene present. Consequently, the naphthalene response approaches that of pure naphthalene. This behavior is similar to a Raoult's Law relationship in which the partial pressure of a liquid is proportional to the mole fraction of that liquid in solution. However, in the case of naphthacene, the addition of pyrene carrier does not produce any significant decrease in the volatility of the already nonvolatile naphthacene particles, so no enhancement is observed.

\section{Conclusions}

Based on these experiments, the carrier effect seems to be largely the result of a combination of particle volatility and surface-area-to-volume ratios. The addition of a nonvolatile carrier can, in certain situations, greatly improve PB-LC/MS sensitivity for volatile (even slightly volatile) analytes. However, the addition of such a carrier seems to be effective only when the carrier is less volatile than the analyte. Also, while the effect of particle mass may play a greater role at lower concentrations (e.g. $<0.1 \mathrm{ng} / \mu \mathrm{L}$ ), the mass of the particles seems to have little effect on transport through the interface within the range of 500pg to $13.5 \mu \mathrm{g}$ injected $(-100-2,800 \mathrm{~nm}$ dry 


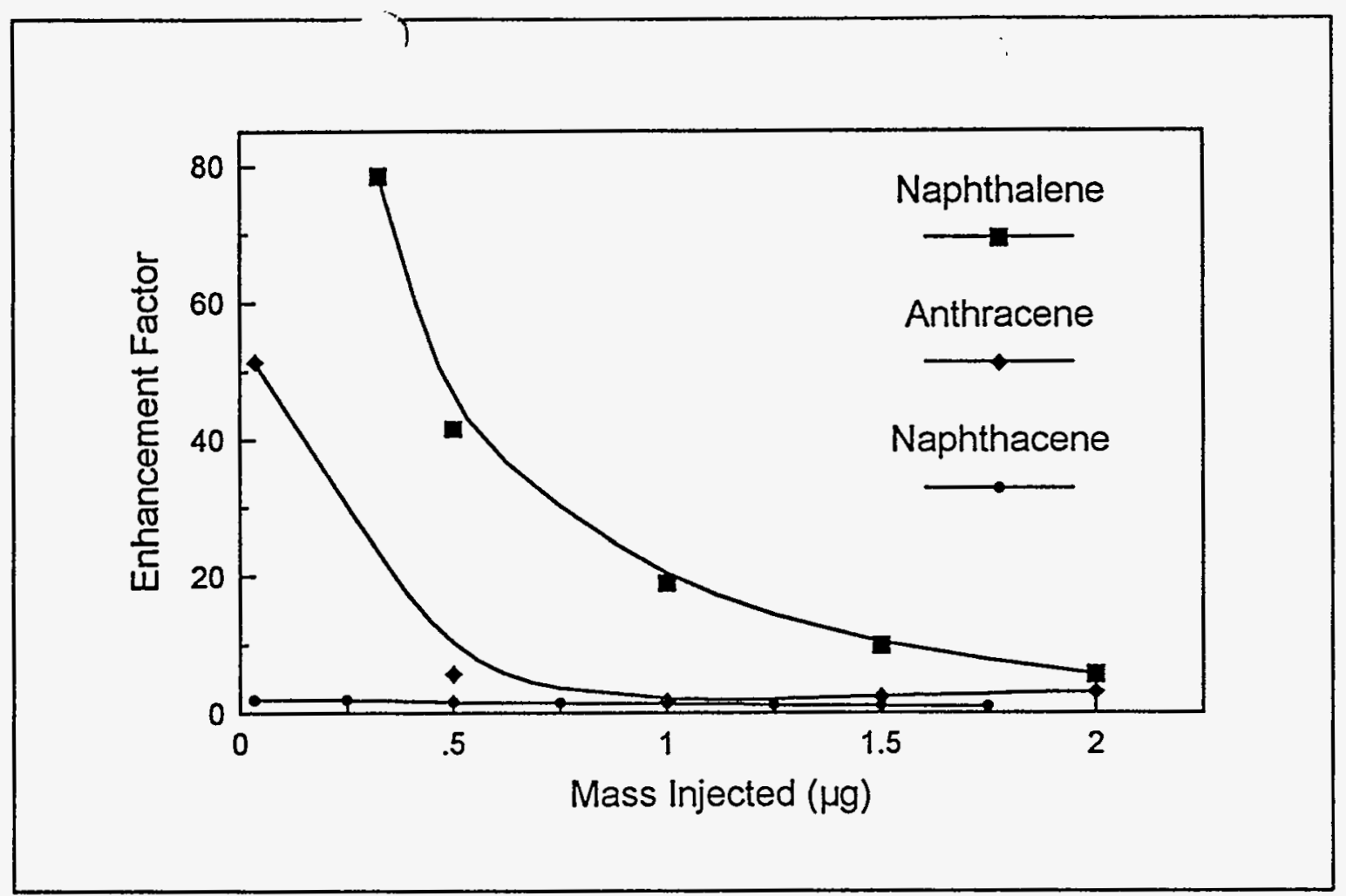

Figure 1.6 Enhancement Factor ws. Amount Injected (13.5 $\mu \mathrm{g}$ Carrier)

particle diameter). In fact, some examples in the literature in which an increase in dry particle mass is used to explain increases in signal, can also be explained on the basis of a decrease in $\mathrm{SA} / \mathrm{V}$ ratios of slightly volatile analytes [9].

It must also be noted that a decrease in analyte volatility is only advantageous up to a point. Highly nonvolatile species are better transported through the interface; however, once inside the mass spectrometer, analyte vaporization must occur before mass analysis can be performed. Tinke et al. reported that of the particles that pass through the momentum separator, only about $10 \%$ are vaporized by collision with the inner walls of the hot ion source [10]. The remaining $90 \%$ strike the inner walls of the transport tube or the outer opening of the ion source. As a result of this gradient, some particles are flash vaporized, some vaporize much more slowly at lower temperatures, and some are not vaporized at all. This phenomenon results in decreased sensitivity, as well as considerable peak broadening. These researchers demonstrated that increased sensitivity and decreased peak broadening of some nonvolatile compounds could be initiated by derivatization to form more volatile compounds [11]. Therefore, both transport and vaporization must be considered when appraising $\mathrm{PB}$ performance. (It should be noted that most newer versions of PB-LC/MS instruments have undergone design improvements in which the momentum separator is positioned much closer to the ion source, with little or no transport tube. This greatly reduces peak broadening and increases sensitivity.)

Concerning quantitation and linearity in PB-LC/MS, the carrier effect will continue to cause unpredictable signal intensities. However, several researchers have demonstrated that accurate quantitation is possible with the use of isotopically labelled internal standards. Difficulties in quantitation are common to almost all LC/MS interfaces, including electrospray and thermospray.

\section{References}


1. Vestal, C.H.; Ferguson, G.J.; Vestal, M.L. Int. J. Mass Spectrom. Ion Processes 1986, 70, 185.

2. Games, D.E.; Alcock, N.J.; Cobelli, L.; Eckers, C.; Games, M.P.L.; Jones, A.; Lant, M.S.; McDowell, M.A.; Rossiter, M.; Smith, R.A.; West, S.A.; Wong, Y. Int. J. Mass Spectrom. and lon Phys. 1983, 46, 81.

3. Canals, A.; Wagner, J.; Browner, R.F.; Hernandis, V. Spectrochim. Acta, Part B 1988, $43,1321$.

4. Scott, R.P.W., Ed., "Small Bore Liquid Chromatography Columns: Their Properties and Uses"; John Wiley and Sons: New York, 1984.

5. Browner, R.F.; Winkler, P.C.; Perkins, D.D.; Abbey, L.E. Microchem. J. 1986, 34, 15.

6. Esmans, E.L.; Geboes, P.; Luyten, Y.; Alderweireldt, F.C. Biomed. Mass Spectrom. 1985, 12, 241.

7. Kresbach, G.M.; Baker, R.R.; Nelson, R.J.; Wronka, J.; Karger, B.L.; Vouros, P. J. Chromatogr. 1987, 394, 89.

8. McFadden, W.H. J. Chromatogr. Sci. 1979, 17, 2.

9. J. S. Ho, T. D. Behymer, W. L. Budde, T. A. Bellar, J. Am. Soc. Mass Spectrom. 3662 (1992).

10. T. A. Bellar, T. D. Behymer, and W. L. Budde, J. Am. Soc. Mass Spectrom. 192 (1990).

11. A. P. Tinke, R. A. M. van der Hoeven, W. M. A. Niessen, U. R. Tjaden, and J. van der Greef, J. Chromatogr. 554119 (1991). 


\section{PUBLICATIONS UNDER PRESENT GRANT DURING REPORT PERIOD}

\section{Reviewed Journals (Citing DOE Support)}

1. "Chromatographic Evaluation of the Particle Beam LC/MS Interface," W.E. Harris, D.D. Perkins and R.F. Browner, Journal of the American Society for Mass Spectrometry, submitted for publication.

2. "Transport Characteristics of the Particle Beam LC/MS Interface," K. Edman and R.F. Browner, Analytical Chemistry, submitted for publication.

3. "Aerosol Properties of the Particle Beam LC/MS Interface," W. Eric Harris and R.F. Browner, Analytical Chemistry, submitted for publication.

\section{Conference Presentations (Citing DOE Support)}

1. "The Next Logical Step: LC/MS in Environmental Analysis," 6th Waldbronner Themen, Enklosterle, West Germany, March 1990 (invited talk).

2. "Particle Beam LC/MS for Environmental Monitoring, "Environmental Teleconference on Newest Analytical Methods and Techniques, San Jose, CA, April 1990 (invited talk).

3. "An Overview of Particle Beam LC/MS," Richard F. Browner, J. Daniel Kirk and W. Eric Harris, 7th (Montreux) Symposium on Liquid Chromatography Mass Spectrometry, Montreux, Switzerland, Nov. 1990 (invited talk).

4. "Aerosol Characterization for ICPAES and ICP/MS," Richard F. Browner, 201st ACS Meeting, Atlanta, GA, April 1991, Peter Keliher Memorial Symposium (invited talk).

5. "Strategies for Increasing the Molecular Weight and Polarity Range of Particle Beam LC/MS," Richard F. Browner, Ohio Valley Chromatography Symposium., Heuston Woods, OH, June 1991 (invited talk).

6. "Particle Beam LC/MS in Environmental Analysis: Considerations for Vaporization and Ionization of Thermally Labile Species," Richard F. Browner and W. Eric Harris, 8th Montreux LC/MS Meeting at Cornell, Cornell, NY, July 1991 (invited talk).

7. "Particle Size Effects In Aerosol Generation, Transport and Vaporization for Particle Beam LC/MS," Richard F. Browner, W. Eric Harris and Katherine Edman, 202nd ACS Meeting, New York, NY, August 1991 (invited talk).

8. "LC/MS Interfacing: Fundamental Studies of Aerosol Formation and Transport," Matthew A. Tarr, Kevin L. Goodner, Brian A. Williams, Guangxuan Zhu and Richard F. Browner, 1992 Pittsburgh Conference on Analytical Chemistry and Applied Spectroscopy, New Orleans, LA, March 1992

9. "Influence of Aerosol and Source Properties on EI, CI and FAB Spectra from Particle Beam LC/MS," Richard F. Browner, W. Eric Harris, J. Daniel Kirk and Jerry C. Richardson, 1992 Pittsburgh Conference on Analytical Chemistry and Applied Spectroscopy, New Orleans, LA, March 1992 (invited talk).

10. "Optimization of Transport Characteristics for the Particle Beam LC/MS Interface," J. Chris Richardson, Katherine R. Edman, W. Eric Harris, and Richard F. Browner, 1992 Pittsburgh Conference on Analytical Chemistry and Applied Spectroscopy, New Orleans, LA, March 1992. 
11. "Influence of Aerosol Drop Size on Response Factors and Linearity in Particle Beam Liquid Chromatography/Mass Spectrometry Interfacing," W. Eric Harris, Katherine Edmans, J. Chris Richardson and Richard F. Browner, American Society for Mass Spectrometry Meeting, Washington, D.C. 1992.

12. "Particle Beam LC/MS Interfacing for Trace Organic Compound Detection," Richard F. Browner, Society for Applied Spectroscopy, Cincinnati Section, April 1992 (invited talk; SAS Tour Speaker).

13. "Strategies for Increasing the Molecular Weight and Polarity Range of Particle Beam LC/MS," Richard F. Browner, Ohio Valley Chromatography Symposium., Heuston Woods, OH, June 1991, (invited talk). 